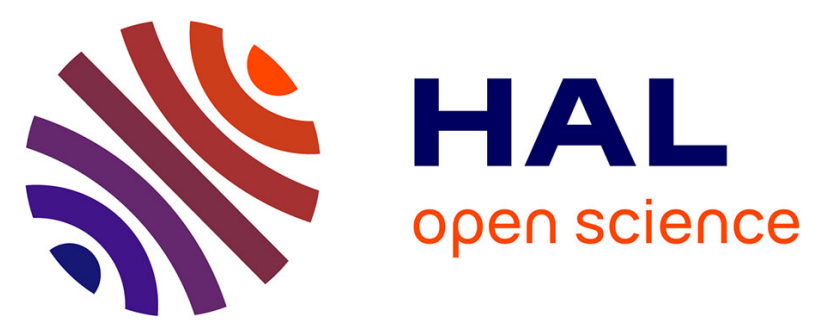

\title{
Nanoscale Spatial Resolution in Far-Field Raman Imaging Using Hyperspectral Unmixing in Combination with Positivity Constrained Super-Resolution
}

Dominik Winterauer, Daniel Funes-Hernando, Jean-Luc Duvail, Said

Moussaoui, Tim Batten, Bernard Humbert

\section{To cite this version:}

Dominik Winterauer, Daniel Funes-Hernando, Jean-Luc Duvail, Said Moussaoui, Tim Batten, et al.. Nanoscale Spatial Resolution in Far-Field Raman Imaging Using Hyperspectral Unmixing in Combination with Positivity Constrained Super-Resolution. Applied Spectroscopy, 2020, 10.1177/0003702820920688 . hal-02563888

\section{HAL Id: hal-02563888 \\ https://hal.science/hal-02563888}

Submitted on 21 Feb 2022

HAL is a multi-disciplinary open access archive for the deposit and dissemination of scientific research documents, whether they are published or not. The documents may come from teaching and research institutions in France or abroad, or from public or private research centers.
L'archive ouverte pluridisciplinaire HAL, est destinée au dépôt et à la diffusion de documents scientifiques de niveau recherche, publiés ou non, émanant des établissements d'enseignement et de recherche français ou étrangers, des laboratoires publics ou privés.

\section{(c) (1) $\$$}

Distributed under a Creative Commons Attribution - NonCommerciall 4.0 International 


\title{
Nanoscale Spatial Resolution in Far-Field Raman Imaging Using Hyperspectral Unmixing in Combination with Positivity Constrained Super-Resolution
}

\author{
Dominik J. Winterauer ${ }^{1,2, *}$, Daniel Funes-Hernando ${ }^{2}$, Jean-Luc Duvail ${ }^{2}$, Saïd Moussaoui ${ }^{3}$, \\ Tim Batten ${ }^{1}$, and Bernard Humbert ${ }^{2}$
}

\begin{abstract}
This work introduces hyper-resolution (HyRes), a numerical approach for spatial resolution enhancement that combines hyperspectral unmixing and super-resolution image restoration (SRIR). HyRes yields a substantial increase in spatial resolution of Raman spectroscopy while simultaneously preserving the undistorted spectral information. The resolving power of this technique is demonstrated on Raman spectroscopic data from a polymer nanowire sample. Here, we demonstrate an achieved resolution of better than $14 \mathrm{~nm}$, a more than eightfold improvement on single-channel image-based SRIR and $25 \times$ better than regular far-field Raman spectroscopy, and comparable to near-field probing techniques.
\end{abstract}

Keywords

Hyperspectral unmixing, super-resolution, digital image restoration, Raman spectroscopy, nanomaterials

\section{Introduction}

Raman spectroscopy is an important characterization tool for nanomaterials and nanocomposites due to its sensitivity to chemical, vibrational, and electronic properties. ' Far-field Raman spectroscopy is an optical technique and its spatial resolution is bounded by the Rayleigh criterion $0.6 \mathrm{I} \lambda / \mathrm{N} A$ $(0.44 \lambda / N A)$ in wide field (confocal) illumination, limiting the scope of its application. ${ }^{\dagger}$ Nanometric resolution in Raman spectroscopy can be achieved by conducting more complex near-field techniques and tip enhanced Raman spectroscopy. $^{2-6}$ The gain in resolution, however, comes at a large experimental cost and has limited applicability to a small range of samples. In addition, the spectral response of the sample material may get distorted by the plasmonic interaction, often in an intricate and nonlinear fashion, which makes chemical analysis of such data challenging. ${ }^{7,8}$

Purely numerical approaches to resolution enhancement come without these drawbacks. Significant resolution enhancement has been achieved using super-resolution image restoration (SRIR) on single band/channel Raman images, ${ }^{9-12}$ which have been improved using a multiband/-channel approach. ${ }^{5,13}$
In this work, we introduce the hyper-resolution (HyRes) framework for spatial resolution enhancement in hyperspectral imaging. HyRes is a combination of hyperspectral unmixing (HU) and SRIR and follows three steps:

I. Factorization of the raw data into spectral signatures and concentration maps* using $\mathrm{HU}$,

2. Independent SRIR of the concentration maps obtained through HU,

3. Remixing the high-resolution concentration maps with the spectral signatures obtained through $\mathrm{HU}$.

'Renishaw plc, Wotton-under-Edge, UK

${ }^{2}$ Institut des Matériaux Jean Rouxel Nantes (IMN), Nantes, France

${ }^{3}$ Laboratoire des Sciences du Numérique de Nantes (LS2N), Nantes, France

*Current address: Bruker Optik GmbH, Rudolf-Plank Str. 27, 76275 Ettlingen, Germany.

Corresponding author:

Dominik J. Winterauer, Renishaw plc, New Mills, Wotton-under-Edge,

Gloucestershire GLI2 8JR, UK.

Email: dominik.winterauer@protonmail.com 
The result is a hyperspectral data cube with enhanced spatial resolution in all single channel images.

The first two steps of the HyRes concept are identical to previous work; ${ }^{13}$ however, in this work, we present a fresh methodology. A method named bounding hyperplanes $(\mathrm{BH})$ is introduced as an efficient $\mathrm{HU}$ tool that yields largely nonnegative concentration maps suitable for SRIR post-processing. $\mathrm{BH}$ is a hybrid method belonging to both the rotation of principal components ${ }^{14,15}$ and the simplex volume ${ }^{16,17}$ family of HU methods.

The SRIR step is performed by interior point least squares (IPLS) with a pixel-wise non-negativity constraint. ${ }^{18}$ The non-negativity constraint ensures that the resulting high-resolution concentration maps can be interpreted as physical/chemical concentrations and also allows for a solution of the ill-conditioned, and potentially ill-posed, image restoration problem without an explicit regularization term. $^{12}$

The utilization of methods that enforce non-negativity in both the HU and SRIR step of HyRes yields physically meaningful single spectra and single-channel images in the high(spatial)-resolution hypercube resulting from HyRes' final remixing step. Analysis of the resulting single-channel images provides a means to quantify the spatial resolution gain brought by HyRes.

As HyRes is a combination of methods from different fields, it can be approached from different perspectives: (i) It generalizes the multi-band approach ${ }^{5}$ to cases where distinct objects on the sample consist of materials whose spectral responses do not necessarily have mutually discriminating peaks but are distinguishable via HU. (ii) Faithful $\mathrm{HU}$ with respect to the chemical components of the sample allows for independent SRIR of all super-images containing spatial information; joint SRIR, ${ }^{19,20}$ and the complexities involved with it, can be avoided. (iii) Pansharpening ${ }^{21-23}$ is not required as high-resolution images are extracted from the hyperspectral data alone. (iv) HyRes appends SRIR to $\mathrm{HU}^{13}$ (v) HyRes is based on super-resolution optical fluctuation imaging $(\mathrm{SOFI})^{24}$ with temporal variations replaced by spectral variations.

The power of the approach and methodology were demonstrated on Raman spectroscopic data from a bundle of nanowires made of poly-(3,4 ethylenedioxythiophene) (PEDOT). ${ }^{25}$ The HyRes methodology of this paper resulted in an order of magnitude better spatial resolution than past works on SRIR of Raman data ${ }^{9-13}$ and a $25 \times$ enhanced spatial resolution when compared to the raw far-field data.

\section{Sample Data}

Sample preparation, acquisition of sample data and the Raman microscope's response function, and single channel super-resolution are described in detail in previous work. $^{12,25}$ The PEDOT nanowires have diameters of
( $100 \pm 10) \mathrm{nm}$. The Raman microscope's response function is a 2D Gaussian with full width half-maximum (FWHM) of

$$
\begin{aligned}
& F W H M_{x}=(476 \pm 12) \mathrm{nm} \\
& F W H M_{y}=(363 \pm 16) \mathrm{nm}
\end{aligned}
$$

in horizontal $\left(F W H M_{x}\right)$ and vertical $\left(F W H M_{y}\right)$ focal plane direction, respectively. The Raman map pixel size was $20 \mathrm{~nm} \times 20 \mathrm{~nm}$. The best minimum resolvable distance achieved by single channel SRIR at $1437 \mathrm{~cm}^{-1}$ Raman shift (Stokes) was II $3.2 \mathrm{~nm} .^{12}$

\section{The Hyperspectral Observation Model}

\section{Hyperspectral Mixing}

The Beer-Lambert absorption law directly translates to the linear mixing model (LMM) for hyperspectral data ${ }^{26}$

$$
\mathbf{X}=\mathbf{S B}
$$

where the columns of $\mathbf{S} \in \mathbb{R}^{L \times p}$ are the spectral signatures at $L$ spectral channels of the $p$ spectral components and the rows of $\mathbf{B} \in \mathbb{R}^{p \times N}$ are the corresponding concentration maps. The resulting hyperspectral data $\mathbf{X} \in \mathbb{R}^{L \times N}$ can be regarded as a collection of either $N$ spectra (at $L$ spectral channels) or $L$ (vectorized) single channel images (with $N$ pixels).

\section{Imaging by a Detector}

The hyperspectral data $\mathbf{X}$ of Eq. 2 is usually detected by an imperfect device of finite resolution. Consequently, the observed single channel images $\mathbf{Y} \in \mathbb{R}^{L \times M}$ result from the underlying ground truth $\mathbf{X}$ (Eq. 2) via filtering by the detector's response function $\mathbf{H} \in \mathbb{R}^{L \times N \times M}$, which is sufficiently described by an affine transformation ${ }^{19,27}$

$$
\mathbf{Y}_{i, j}=\sum_{k=1}^{N} \mathbf{X}_{i, k} \mathbf{H}_{i, k, j}+\mathbf{E}_{i, j}
$$

where the error term $\mathbf{E} \in \mathbb{R}^{L \times M}$ accounts for any nondeterministic detector response occurring while measuring. As the detector's response function is allowed to vary between different spectral channels, we resort to an index notation for clarity. ${ }^{\S}$

Plugging Eq. 2 into Eq. 3 then yields

$$
\mathbf{Y}_{i, j}=\sum_{k=1}^{N} \sum_{l=1}^{p} \mathbf{S}_{i, l} \mathbf{B}_{l, k} \mathbf{H}_{i, k, j}+\mathbf{E}_{i, j}
$$


The consequences of Eq. 4 are quite far-reaching. The spectral dependency of the spatial detector response introduces an additional mixing between the spectral and spatial degrees of freedom beyond the LMM of Eq. 2. In its most general form, Eq. 4 is prohibitive of the HyRes approach introduced in the introduction as $\mathrm{HU}$ of such a model will become either too computationally costly or too inaccurate.

In the specific case of Raman spectroscopic data, however, one can assume that

$$
\mathbf{H}_{i, j, k} \approx \overline{\mathbf{H}}_{j, k} \forall i
$$

i.e. the spatial detector response is approximately identical for all spectral channels (see Supplemental Material for details). If the assumption Eq. 5 holds, Eq. 4 becomes

$$
\mathbf{Y}_{i, j}=\sum_{l=l}^{p} \mathbf{S}_{i, l} \sum_{k=1}^{N} \mathbf{B}_{l, k} \overline{\mathbf{H}}_{k, j}+\mathbf{E}_{i, j}
$$

and the effects of hyperspectral mixing and imaging commute. By setting

$$
\mathbf{C}=\mathbf{B} \overline{\mathbf{H}}
$$

and dropping indices, Eq. 6 becomes

$$
\mathbf{Y}=\mathbf{S C}+\mathbf{E}
$$

which is the standard form of the LMM found in analytical chemistry, remote sensing, and many other fields concerned with multivariate data processing. ${ }^{17,28}$

The observation model Eq. 6 allows for a straightforward application of the HyRes scheme. $\mathbf{S}$ and $\mathbf{C}$ of Eq. 8 can be estimated via blind linear hyperspectral unmixing (BLHU). Estimates for B can be obtained via SRIR of the estimates $\mathbf{C}$ and in combination with an estimate of $\mathbf{S}$ an estimate of $\mathbf{X}$ can be found.

\section{Hyperspectral Unmixing with Bounding Hyperplanes}

\section{The Rotation Ambiguity}

Given an observation model for a hyperspectral data set, $\mathrm{HU}$ is concerned with estimating the free parameters of the observation model. For the LMM of Eq. 8, the free parameters are the characteristic spectral signatures $\mathbf{S}$, the concentration maps $\mathbf{C}$, and potentially the number of materials $p$. BLHU is $\mathrm{HU}$ of an LMM without knowledge of both $\mathbf{S}$ and $\mathbf{C}$.

An intriguing property of BLHU is that given a pair of estimates $\widehat{\mathbf{S}}$ and $\widehat{\mathbf{C}}$ another pair of estimates $\widehat{\mathbf{S}}^{\prime}$ and $\widehat{\mathbf{C}}^{\prime}$ can be found with the help of an arbitrary invertible $p \times p$ matrix $\mathbf{R}$

$$
\begin{aligned}
& \widehat{\mathbf{S}}^{\prime}=\widehat{\mathbf{S}} \mathbf{R}^{-1} \\
& \widehat{\mathbf{C}}^{\prime}=\mathbf{R} \widehat{\mathbf{C}}
\end{aligned}
$$

Multiplying the new estimates yields

$$
\begin{aligned}
& \widehat{\mathbf{S}}^{\prime} \widehat{\mathbf{C}}^{\prime}=\widehat{\mathbf{S}} \mathbf{R}^{-1} \mathbf{R} \widehat{\mathbf{C}} \\
& =\widehat{\mathbf{S C}}
\end{aligned}
$$

so the pairs $\widehat{\mathbf{S}}$ and $\widehat{\mathbf{C}}$, and $\widehat{\mathbf{S}}^{\prime}$ and $\widehat{\mathbf{C}}^{\prime}$ are equivalent descriptors of the observation $\mathbf{Y}$ of Eq. 8. The rotation ambiguity Eqs. $10 \mathrm{a}$ and $10 \mathrm{~b}$ allow for uncountably infinite optimal estimators for the LMM of Eq. 2 which is the reason behind the vast number of methods, even of classes of methods, for BLHU. ${ }^{29,30}$

While Eqs. IOa and I0b are prohibitive of a unique least squares optimal solution, it is extremely useful from a practical point of view. Given any pair of estimates $\widehat{\mathbf{S}}$ and $\widehat{\mathbf{C}}$, a new pair of estimates $\widehat{\mathbf{S}}^{\prime}$ and $\widehat{\mathbf{C}}^{\prime}$ with the same predictive power can be found. The new estimates $\widehat{\mathbf{S}}^{\prime}$ and $\widehat{\mathbf{C}}^{\prime}$ might then show desirable properties such as non-negativity not exhibited by the original estimates $\widehat{\mathbf{S}}$ and $\widehat{\mathbf{C}}$.

\section{Dimensional Reduction Using Principal Component Analysis}

The first step in the method of $\mathrm{BH}$ is to find an initial pair of optimal estimators $\widehat{\mathbf{S}}$ and $\widehat{\mathbf{C}}$. Principal component analysis (PCA) yields a least squares optimal low-rank approximation to the observed hyperspectral data matrix $\mathbf{Y}^{31-33}$ If the elements of the error term $\mathbf{E}$ are zero-mean independently and identically distributed (i.i.d.) with finite variance, then PCA also yields a least square optimal estimation of the subspace spanned by $\mathbf{S C}$ in Eq. 2 for any given rank $p .{ }^{34}$ PCA for BH calculates the eigenvectors $\left\{\widehat{\mathbf{s}}_{i}\right\}$ and -values $\left\{\lambda_{i}\right\}$ of $\mathbf{Y} \mathbf{Y}^{T}$. There is some debate whether to mean-center the data $\mathbf{Y}$ or not. For details, we refer to the literature. ${ }^{33}$

The scores and loadings returned by PCA constitute the initial estimates $\widehat{\mathbf{S}}$ and $\widehat{\mathbf{C}}$, respectively. Further, the variance explained allows for an estimate of the rank $p$ of the data matrix $\mathbf{Y}$, the number of components.

Figure I shows the linear and logarithmic variance explained for each component in decreasing order. As the variance explained by discriminatory components can be orders of magnitude smaller than the variance explained by common factors, a plot of the logarithmic eigenvalues (Fig. Ib) is often more revealing than a plot of the bare values (Fig. Ia). A simple heuristic to estimate the rank $p$ is then to observe which logarithmic eigenvalues are significantly above a potentially linear baseline. ${ }^{35,36}$ For the present data, there is a clearly visible cut-off at $p=2$ (Fig. Ib). 
(a)

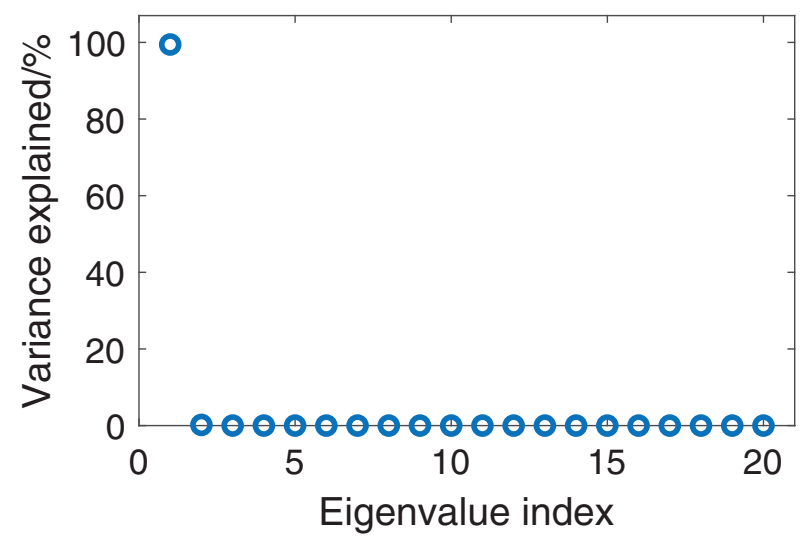

(b)

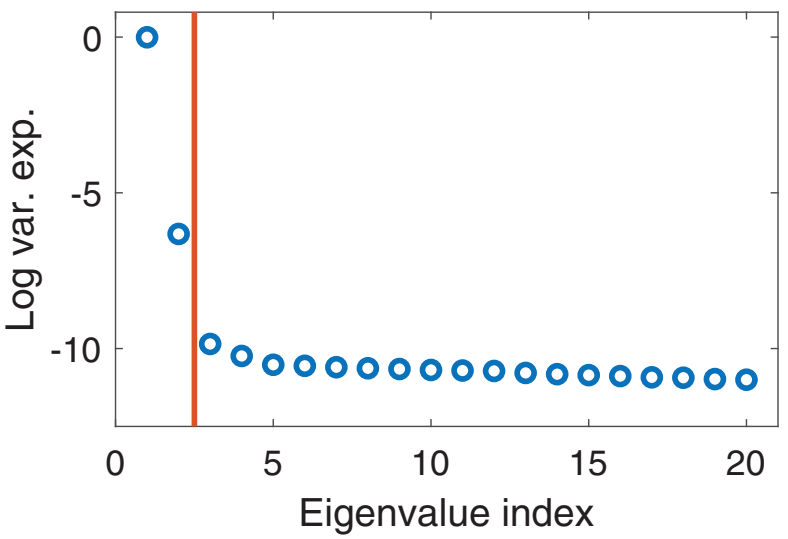

Figure I. Eigenvalue spectrum for $\mathbf{Y} \mathbf{Y}^{T}$ : The (a) bare and (b) logarithmic eigenvalues are plotted in decreasing order of magnitude. If the data $\mathbf{Y}$ has rank $p$, the eigenvalue spectrum should be flat for indices $>p$. Because the variance explained of common and discriminatory factors can vary by orders of magnitude, the true cut-off index is better visible on a logarithmic scale (b).

For more elaborate methods of rank estimation, we refer to the literature. ${ }^{34}$

\section{Rotation of Principal Components by $\mathrm{BH}$}

The loadings $\widehat{\mathbf{S}}$ and scores $\widehat{\mathbf{C}}$ estimated by PCA are uncorrelated, i.e. the columns of $\widehat{\mathbf{S}}$ are mutually orthogonal and so are the rows of $\widehat{\mathbf{C}}$. While uncorrelatedness may be desirable from a statistical point of view, physical models of hyperspectral data are normally only meaningful if $\widehat{\mathbf{C}} \geq 0$ is satisfied.

The biplot in Fig. 2a showing the first and second PCA scores reveals that the first score values are largely nonnegative, while the second score values are distributed symmetrically around zero. Similarly, the first PCA loading can be interpreted as a PEDOT Raman spectrum, while the second PCA loading is hard to interpret on its own, Fig. 2 b.

Simplex geometry considerations for hyperspectral (un-)mixing state that linearly mixed hyperspectral data with $p$ linearly independent spectral components must be confined to a $p$ simplex, with one vertex being the darkpoint of the instrument and the other $p$ vertices the spectral signatures of the constituent components. ${ }^{16}$ The simplex structure remains intact after transformation and dimensional reduction, given that the resulting space is not orthogonal to any of the constituent spectral signatures. ${ }^{\dagger \dagger}$

The scores in the biplot Fig. $2 \mathrm{a}$ are indeed confined to a two-simplex, i.e. a triangle, with the vertex at the origin being the dark-point of the Raman microscope. The position of the spectral signatures can be found by exploiting the simplex structure of the scores. The spectral signatures are along the sides of the enclosing triangle that pass through the origin. Simplex volume techniques such as $n$-FINDR, ${ }^{37}$ vertex component analysis, ${ }^{38}$ and simplex identification via split augmented Lagrangian ${ }^{39}$ recover the simplex vertices directly, whereas simplex facet techniques such as hyperplane-based Craig-simplex identification (HyperCSI) ${ }^{40}$ and facet component analysis ${ }^{4 I}$ recover the vertices via the facets of the simplex enclosing the data. These techniques ${ }^{37-41}$ have been developed specifically for sum-to-one constrained data, i.e.,

$$
\mathbf{C}^{T} \mathbf{I}_{p}=\mathbf{I}_{N}
$$

where $\mathbf{I}_{k}$ denotes the $k$-dimensional column vector with all entries equal to I. If a sum-to-one constraint does not hold, it has to be enforced via projection, which either increases the noise level in the data or decreases the number of data points on the simplex surface. ${ }^{38,41}$

$\mathrm{BH}$ is specifically for data that are not sum-to-one constrained. It finds the $p$ hyperplanes through the origin (darkpoint) bounding the data after dimensional reduction to $p$ dimensions. The net effect is that the coordinate system is rotated such that the new coordinate axes are aligned with the bounding hyperplanes (see Figs. $2 \mathrm{a}$ and $2 \mathrm{c}$ ). This defines $\mathbf{R}$ of Eq. $9 b$ up to an arbitrary linear scaling which is chosen such that $\widehat{\mathbf{C}}^{\prime} \preceq$ I. With a proper choice of sign, the bulk of the rotated scores will be transformed to the nonnegative orthant (Fig. 2c).

Both the presence of noise and small deviations from exact linear mixing (Eq. 2) push some scores outside of the ideal bounding simplex. ${ }^{38}$ Consequently, one has to allow for $-\epsilon \preceq \widehat{\mathbf{C}}^{\prime}$ for an $\epsilon>0$ and search for hyperplanes that are not strictly bounding the data. ${ }^{39-41} \mathrm{BH}$ uses a noise-robust method for fluorescence background removal for bounding hyperplanes identification. ${ }^{42}$ In turns, each of the $p$ reduced dimensions is assumed to be a quantity dependent on the remaining $p-I$ dimensions. Finding upper and lower bounding linear backgrounds in the $p-I$ independent dimensions then identifies the bounding hyperplanes (Fig. 2a). 
(a)

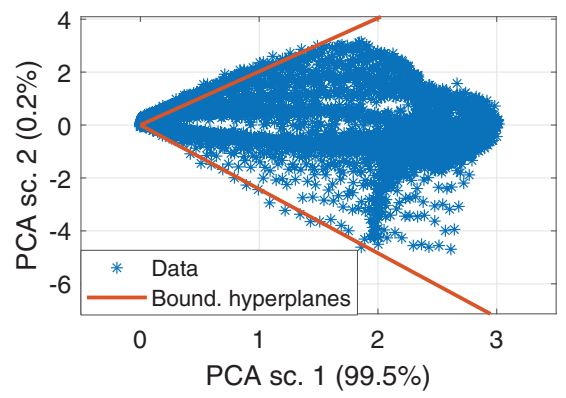

(c)

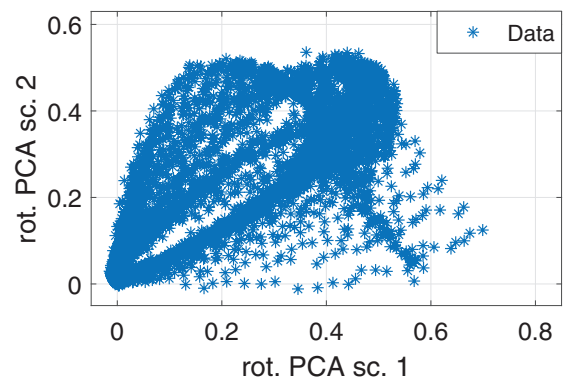

(b)

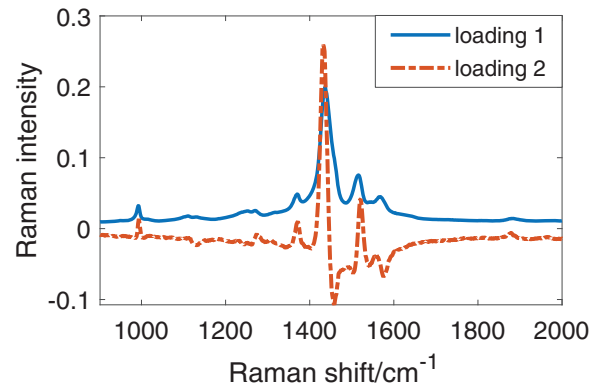

(d)

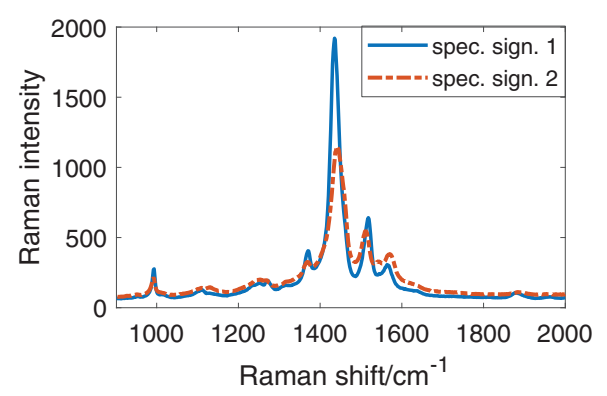

Figure 2. Hyperspectral data after PCA. (a) The second score plotted against the first score for each data point/spectrum. The plot has been rescaled to unit variance along each axis for better visibility, the variance explained is given in parenthesis. The bounding hyperplanes to the data cloud are indicated. (b) The first and second PCA loading (unit Euclidean norm). (c) Rotated PCA scores plotted against each other after an oblique rotation. The bounding hyperplanes of Fig. $2 \mathrm{a}$ are the new coordinate axes. The rotated scores are rescaled such that they are $\leq$ I. (d) Rotated PCA loadings.

The spectral signatures resulting from $\mathrm{BH}$ shown in Fig. $2 \mathrm{~d}$ are both non-negative and each can be interpreted as a variant of PEDOT spectra, ${ }^{43}$ which is a strong indication that the spectral signatures were estimated faithfully.

\section{BH Pre- and Post-Processing}

For dimensional reduction, $\mathrm{BH}$ resembles methods from the rotation of PCA family, ${ }^{14,44}$ but $\mathrm{BH}$ can also be used in conjunction with other dimensional reduction techniques such as maximum noise fraction ${ }^{45}$ or hyperspectral subspace identification by minimum error (HySime). ${ }^{46}$

For a strict enforcement of nonnegativity, the BLHU results $\widehat{\mathbf{S}}^{\prime}$ and $\widehat{\mathbf{C}}^{\prime}$ from $\mathrm{BH}$ can be post-processed with nonnegative matrix factorization (NMF) techniques (possibly at the expense of least-squares optimal data fidelity). ${ }^{47-52}$

\section{Super-Resolution}

Since only estimates $\widehat{\mathbf{C}}^{\prime}$ of $\mathbf{C}$ are available, the LMM of Eq. 7 has to be slightly modified to include an estimation error $\mathbf{E}^{\prime}$ in the SRIR forward model

$$
\widehat{\mathbf{C}}^{\prime}=\mathbf{B} \overline{\mathbf{H}}+\mathbf{E}^{\prime}
$$

Further, we require that obtained estimates $\widehat{\mathbf{B}}$ for $\mathbf{B}$ fulfill $\widehat{\mathbf{B}} \succeq 0$. The sought-after estimate $\widehat{\mathbf{B}}$ is then the solution of the following SRIR optimization problem

$$
\widehat{\mathbf{B}}=\arg \min _{\mathbf{B}}\left\|\widehat{\mathbf{C}}^{\prime}-\mathbf{B} \overline{\mathbf{H}}\right\|_{F}^{2}
$$$$
\text { s.t. } \quad \mathbf{B} \succeq 0
$$

Equation I $3 \mathrm{~b}$ can be solved for each of the $p$ rows $\mathbf{B}_{i, \text { : }}$ of B independently, i.e. in parallel, using IPLS. IPLS is an iterative method that performs a full or partial Newton step in the primal variable $\mathbf{B}_{i, \text { a }}$ and a dual variable $\lambda \in \mathbb{R}^{N}$ towards the perturbed Karush-Kuhn-Tucker optimality conditions

$$
\begin{aligned}
& 2 \overline{\mathbf{H}}\left(\widehat{\mathbf{C}}_{i,:}^{\prime}-\mathbf{B}_{i, i} \overline{\mathbf{H}}\right)^{T}+\lambda=\mathbf{0} \\
& \lambda \circ \mathbf{B}_{i,:}=\mu_{k} \mathbf{I} \\
& \mathbf{B}_{i,:} \geq \mathbf{0} \\
& \lambda \geq \mathbf{0}
\end{aligned}
$$

for a perturbation parameter $\mu_{k}>0$ at iteration $k^{*} \mu_{k}$ is updated at each iteration in a non-increasing fashion such that $\mu_{k} \rightarrow 0$ as $k \rightarrow \infty$. Details on how to determine the detector response $\overline{\mathbf{H}}$ and how to implement IPLS can be found in previous work. ${ }^{12,18}$ 


\section{Results}

\section{SRIR of Concentration Maps}

The concentration maps shown in Fig. 3 were superresolved via IPLS. SRIR was performed for upsampling factors of $1 \times 1$ and $2 \times 2$ resulting in pixel sizes of $20 \mathrm{~nm} \times 20 \mathrm{~nm}$ ('20 nm' for brevity) and $10 \mathrm{~nm} \times 10 \mathrm{~nm}$ ('IO nm' for brevity), respectively. Upsampling does not necessarily enhance resolution, but it increases the sharpness of the resulting image, as becomes apparent by comparing images from Fig. 4 with different pixel sizes.

The sharpness of a super-resolved image is only a lower bound to, but no estimator for, the minimum resolvable distance. ${ }^{12}$ It can become a source of high resolution when multiple super-resolved images are combined into a single high-resolution image.

The super-resolved images of the concentration maps $\widehat{\mathbf{C}}_{1,:}^{\prime}$ and $\widehat{\mathbf{C}}_{2,:}^{\prime}$ in Fig. 4 show a nanowire-like structure each which is very desirable for data from a nanowire sample. An AFM image of the sample area, which is shown in Fig. $4 \mathrm{~b}$ of previous work, ${ }^{12}$ indicates that two PEDOT nanowires cross the sample area. Therefore, unmixing two chemical components whose high-resolution concentration maps show nanowire-like structures is both satisfying and reassuring.

The super-resolved concentration maps of the second component $\widehat{\mathbf{C}}_{2, .}^{\prime}$, Figs. $4 \mathrm{~b}$ and $4 \mathrm{~d}$, also show a little feature set apart from the nanowire-like structure. Such a feature can arise from a PEDOT nanoparticle, e.g. a nanowire debris, or a local deformation of the nanowire that is interpreted as a separate feature by SRIR. Further, it may also arise from interactions between the two nanowires, although this would result in additional spectral components rather than SRIR artifacts.

\section{Remixing the High-Resolution Data}

The high-resolution concentration maps in Fig. 4 represent the estimate $\widehat{\mathbf{B}}$ of the high-resolution concentration maps $\mathbf{B}$ in the hyperspectral imaging model of Eq. 6 . An estimate $\widehat{\mathbf{X}}$ of the high-(spatial)-resolution hyperspectral data cube $\mathbf{X}$ can be recovered via Eq. 2

$$
\widehat{\mathbf{X}}=\widehat{\mathbf{B}} \widehat{\mathbf{S}}^{\prime}
$$

Like the low-resolution hyperspectral data cube, the high-resolution data cube can be sliced in the spectral domain to obtain high-resolution single channel gray-scale images. Choosing the channel of maximum intensity at $1437 \mathrm{~cm}^{-1}$ (Fig. 2d) results in the images shown in Fig. 5.

\section{Estimation of Achieved Resolution}

The resolution in Figs. $5 \mathrm{a}$ and $5 \mathrm{~b}$ can be inferred by inspecting the sections labeled $\mathrm{SI}$ and S2 across both nanowire components shown in Fig. 5. The minimum distance at which the nanowires remain distinguishable is then identified as the minimum resolvable distance. Since the nanowires have finite width, we use their edge separation as a distance measure for resolution purposes. Because their edges are blurred, we define them to be at $\mathrm{I} / \mathrm{e} \approx 0.368$ times the respective peak intensities. (a)

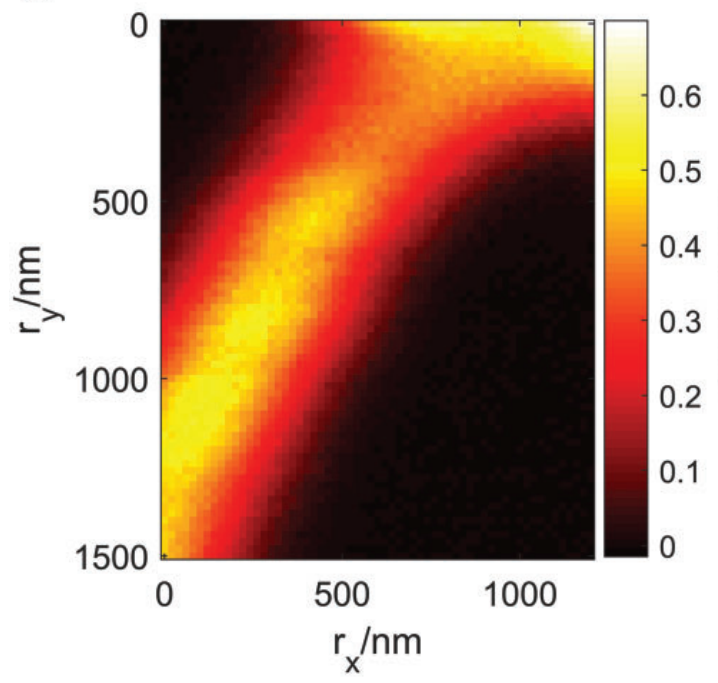

(b)

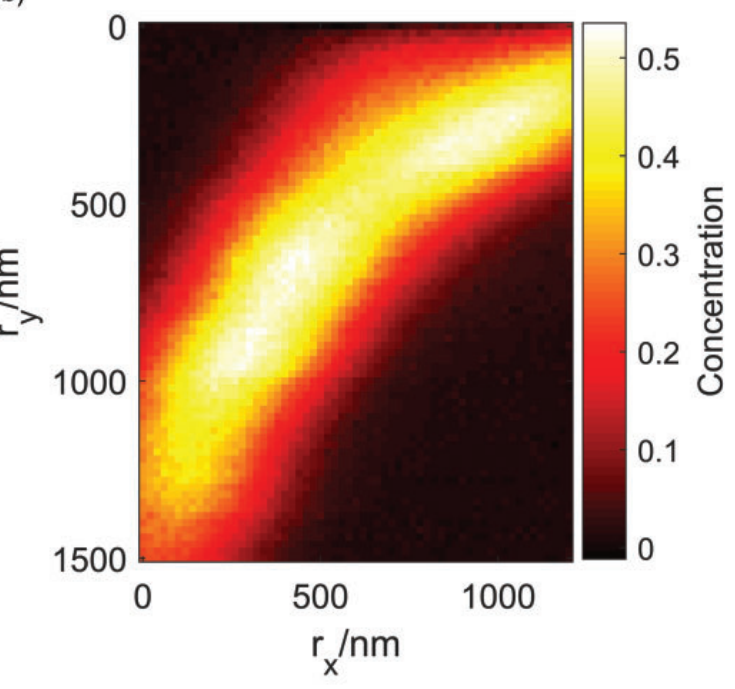

Figure 3. Images of concentration maps: (a) Distribution of first $\left(\widehat{\mathbf{C}}_{1, .}^{\prime}\right)$ and (b) second $\left(\widehat{\mathbf{C}}_{2, .}^{\prime}\right)$ rotated PCA scores on sample area. The two concentration maps were extracted from a single hyperspectral data set. 
(a)

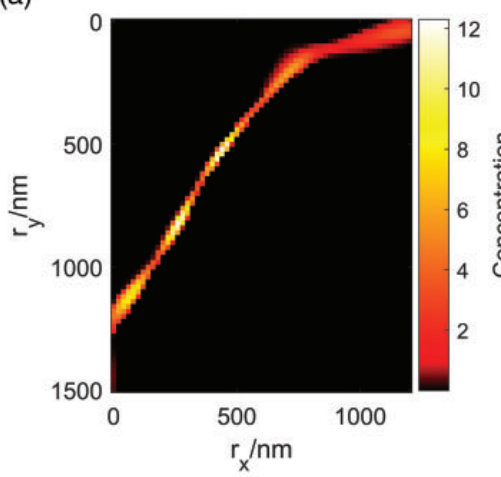

(c)

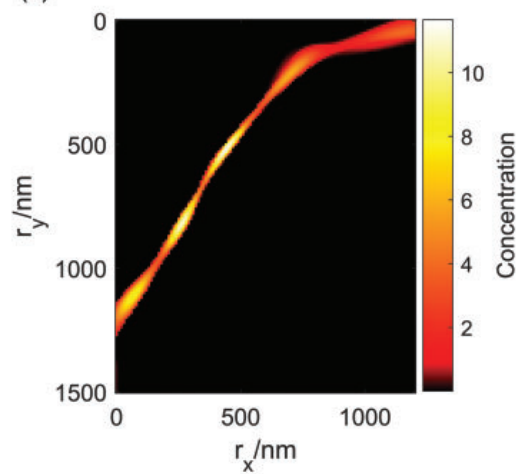

(b)

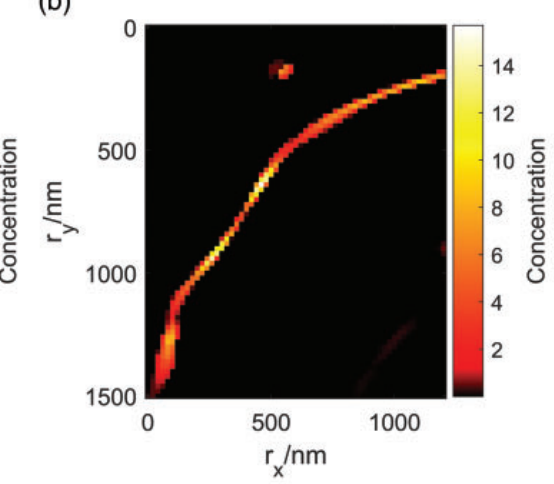

(d)

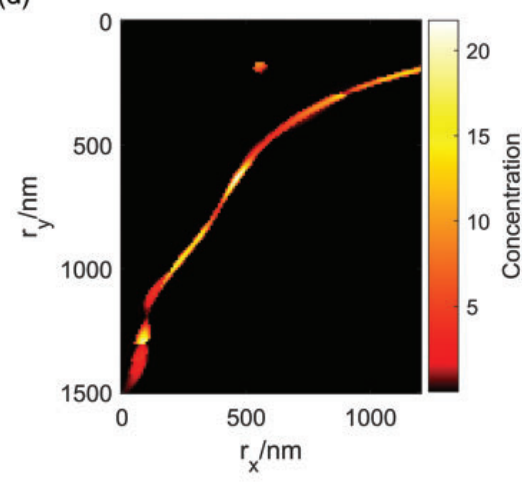

Figure 4. Super-resolution of the Raman heat maps shown in Fig. 3 for resulting pixel sizes of (a,b) $20 \mathrm{~nm}$ and (c,d) $10 \mathrm{~nm}$. While the super-resolved images of $\widehat{\mathbf{C}}_{1 ;}^{\prime}(\mathrm{a}, \mathrm{c})$ show only a nanowire-like structure, the super-resolved images of $\widehat{\mathbf{C}}_{2 ;}^{\prime}$ (b,d) show an additional feature. The feature could either be local contamination with a PEDOT debris or an artifact of SRIR caused by a local deformation of the respective PEDOT nanowire.
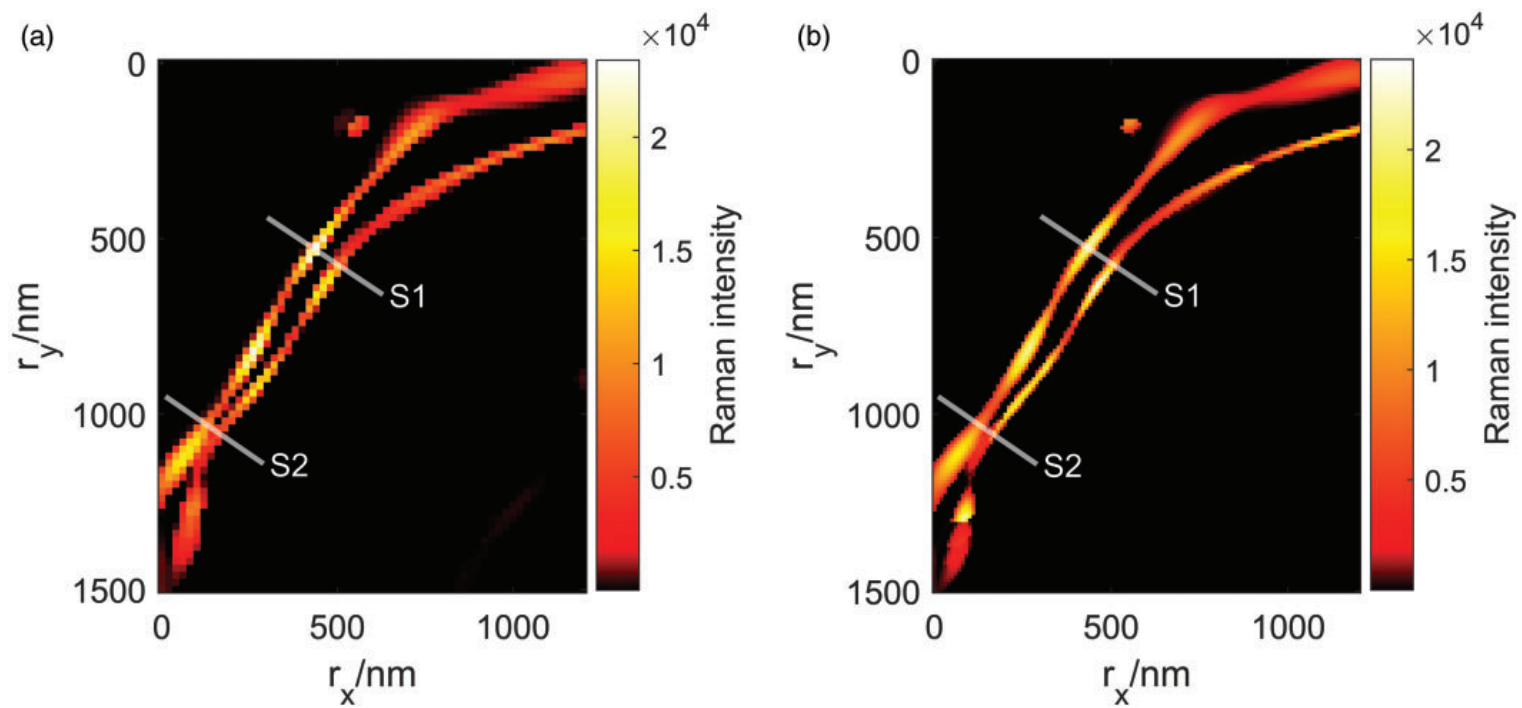

Figure 5. Recombined super-resolved images at $1437 \mathrm{~cm}^{-1}$ for (a) $20 \mathrm{~nm}$ and (b) $10 \mathrm{~nm}$ resulting pixel size. The recombined images are obtained via a weighted sum of Figs. $4 \mathrm{a}$ and $4 \mathrm{~b}$, and Figs. $4 \mathrm{c}$ and $4 \mathrm{~d}$, respectively. The weights are given by the intensity of the respective rotated loading (Fig. $2 \mathrm{~d}$ ) at the given wavenumber. Line profiles along the sections SI and S2 for both figures are shown in Fig. 6. 
The smallest resolvable distance can be found along section S2. While the two nanowires are unresolvable in the $20 \mathrm{~nm}$ image, they are clearly resolvable in the $10 \mathrm{~nm}$ image, with an edge separation of less than $14 \mathrm{~nm}$, below the pixel size of the raw data. If the component information was retained during remixing, distinct objects could be resolved even if they overlap, pushing the resolvable distance de facto to $0 \mathrm{~nm}$ (see Fig. 6b).

\section{HyRes Lack of Fit}

Although there is no ground truth to compare against, at least the lack of fit (LoF) within the model Eq. 6 and its substeps Eq. 8, and Eqs. 7 and 12 can be computed. The LoF resulting from $\mathrm{HU}$ is

$$
\begin{aligned}
& \operatorname{LoF}_{H U}=\left\|\mathbf{Y}-\widehat{S}^{\prime} \widehat{C}^{\prime}\right\|_{F} /\|\mathbf{Y}\|_{F} \\
& \approx 6.1 \%
\end{aligned}
$$

where $\|\cdot\|$ is the Frobenius norm. The LoF from SRIR is

$$
L o F_{S R I R}=\left\|\widehat{\mathbf{C}}^{\prime}-\widehat{\mathbf{B}} \overline{\mathbf{H}}\right\|_{F} /\left\|\widehat{\mathbf{C}}^{\prime}\right\|_{F}
$$

and is found to be

$$
\begin{aligned}
& L_{0} F_{S R I R, I} \approx 7.1 \% \\
& \operatorname{LoF}_{S R I R, 2} \approx 7.2 \%
\end{aligned}
$$

for upsampling factors of $\mathrm{I}\left(\right.$ LoF $\left._{\mathrm{SRIR}, \mathrm{I}}\right)$ and 2 (LoF $\left.\mathrm{F}_{\mathrm{SRIR}, 2}\right)$, respectively. The LoF for the total HyRes procedure is then computed by

$$
L o F_{\text {tot }}=\left\|\mathbf{Y}-\widehat{\mathbf{S}^{\prime}} \widehat{\mathbf{B}} \overline{\mathbf{H}}\right\|_{F} /\|\mathbf{Y}\|_{F}
$$

and is

$$
\begin{aligned}
& L_{o F_{\text {tot, }}} \approx 8.4 \% \\
& \text { LoF }_{\text {tot, } 2} \approx 8.5 \%
\end{aligned}
$$

for upsampling factors of $\mathrm{I}\left(\operatorname{LoF}_{t o t, 1}\right)$ and 2 (LoF $\left.F_{t o t, 2}\right)$, respectively.

\section{Discussion}

\section{Hyperspectral Unmixing: A Great Source of Resolving Power}

In microscopy, resolution is the minimum separation between two distinct objects at which they are perceived as separate. The resolving power of the microscope stems from the proper alignment of light sources, mirrors, and lenses, which then allows the observer to distinguish objects that would not be distinguishable by the naked eye. The equivalent source of resolving power in HyRes is $\mathrm{HU}$, as it distinguishes separate objects that may or may not be distinguishable in any of the $L$ single channel images obtained from the sample. Two objects, or two (a)

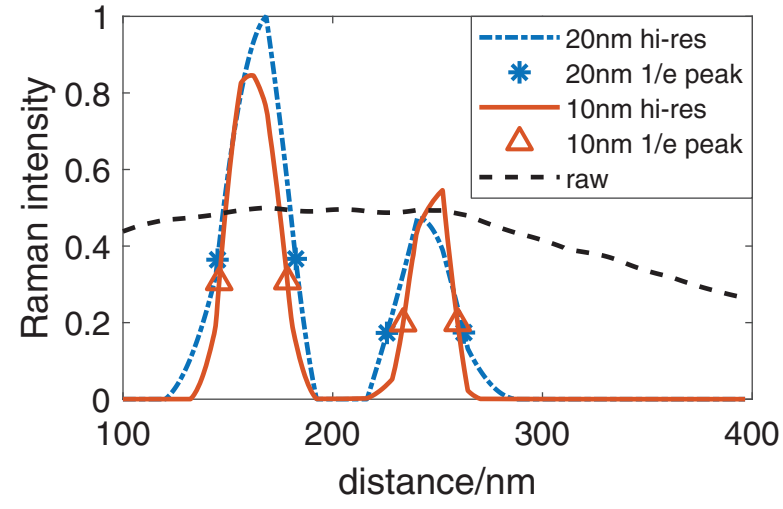

(b)

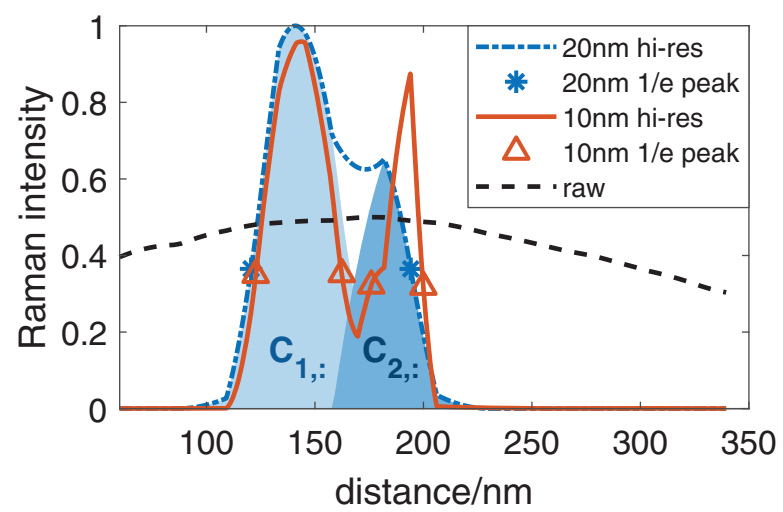

Figure 6. Intensity line profiles along image sections of Fig. 5: The sections range across both nanowires whose edges are assumed at I/e times the peak intensity and indicated by stars ( $20 \mathrm{~nm}$ step) and triangles ( $10 \mathrm{~nm}$ step). The line profiles were obtained through linear interpolation of the respective images. (a) Intensity line profile along section SI: Two objects are resolved, each of them appearing as a peak. The two peak maxima are separated by $70.7 \mathrm{~nm}(20 \mathrm{~nm}$ step) and $62.9 \mathrm{~nm}$ ( $10 \mathrm{~nm}$ step). The distance between the edges of the two objects is $25.6 \mathrm{~nm}$ and $29.6 \mathrm{~nm}$, respectively. (b) Intensity line profile along section S2: The two peak maxima are separated by $40.9 \mathrm{~nm}(20 \mathrm{~nm}$ step) and $50.3 \mathrm{~nm}$ ( $10 \mathrm{~nm}$ step). The two objects are resolved in the $10 \mathrm{~nm}$ pixel size image with an edge separation of $13.6 \mathrm{~nm}$. In the $20 \mathrm{~nm}$ pixel size image, the two objects are unresolved unless the contributions from different components are tagged $\left(\mathbf{C}_{1}\right.$ and $\left.\mathbf{C}_{2,:}\right)$, in which case the objects remain distinguishable. 
parts of the same object, whose spectral signatures differ sufficiently relative to the noise level in the data, can be identified via $\mathrm{HU}$ at arbitrary separation given that their projections on the focal plane are not congruent. The resolving power of $\mathrm{HU}$ is thus infinite in theory, although perhaps limited by the pixel size in practice. The resulting concentration maps in Fig. 3 are as blurry as the raw single channel image, but as far as resolution is concerned, all that matters is the distinction of (separate) objects (components I and 2) which has clearly been achieved.

\section{High-Resolution Images Using SRIR}

Resolution enhancement by $\mathrm{HU}$ alone would call for a statistical criterion of resolution or, rather, separability replacing the Rayleigh criterion. Moreover, the achieved resolution might be hard to visualize or quantify from the images in Fig. 3 alone. SRIR allows to merge the distinct low-resolution concentration maps into a high-resolution hyperspectral data cube for which Rayleigh's and similar resolution criteria can be straightforwardly applied to each single channel image. A large degree of sharpness in the super-resolved concentration maps proves to be key to high-resolution single-channel images in the remixed high-resolution hyperspectral data cube. The achieved resolvable distance of less than $14 \mathrm{~nm}$ is more than a factor 8 better, i.e. smaller, than the less than I I $4 \mathrm{~nm}$ achieved by single channel image-based SRIR on the same data and more than a factor 25 better than the intrinsic resolution of the confocal Raman microscope.

The resolution enhancement stems from the resolving power offered by $\mathrm{HU}$ and exclusively arises between objects that belong to different chemical compounds. The intra-component achievable resolution is unaffected by $\mathrm{HU}$ and thus remains limited by the single channel image SRIR bounds reported previously. ${ }^{9-12}$

\section{The Role of Bounding Hyperplanes}

While the resolving power of $\mathrm{HU}$ is fairly unconditional, generating high-resolution images from $\mathrm{HU}$ concentration maps is not. The ability to generate high-resolution images, Fig. 5, depends strongly on an adequate estimation of the non-negative concentration maps, Fig. 3. Any residual mixing can show up as artifacts (both spatial and spectral) in the super-resolved hyperspectral data cube which potentially prohibits a visualization of the resolution gain provided by HU. Further, if multiple spectral components are required to reconstruct the proper concentrations in congruent areas, one has to resort to joint image restoration techniques that are usually computationally more expensive than independent restoration. A well enough estimation of the non-negative concentration maps $\mathbf{C}$ is not exclusive to $\mathrm{BH}$. NMF techniques often yield comparable results. For very noisy data, however, strict non-negativity constraints introduce unnecessary artifacts and soft constraints as in $\mathrm{BH}$ become preferable. ${ }^{39,53}$

\section{Improved Spectral Resolution}

As the roles of scores and loadings can be interchanged, ${ }^{48}$ HyRes can also be seen as a framework to improve spectral resolution, and Eq. 5 implies that the spectral distortion is (almost) identical for each pixel. If Eq. 5 holds in both the spectral and the spatial domain, spectral and spatial resolution enhancement can be performed simultaneously and independently after the HU step. The resulting remixed hyperspectral data cube has then enhanced spectral and spatial resolution.

\section{Measurement and Computation Time}

Data acquisition of the far-field Raman data took $\sim 62$ min. The overhead emerging from HyRes included the acquisition of the microscope's PSF along two directions (I min each) and the computation time required for HyRes, which was about I min for an upsampling factor of I and a little less than 3 min for an upsampling factor of 2 using Matlab (The Mathworks, Inc.) and its parallel processing toolbox on a multi-core laptop. Thus, with a little less than $5 \mathrm{~min}$, total overhead the resolution was enhanced more than 25 -fold.

If the condition of the instrument remains stable within a series of measurements, then the PSF measurement needs to be carried out only once, further reducing the overhead per measurement.

\section{Nanometric Resolution and Accuracy of Results}

The potential for nanometric resolution in far-field Raman imaging derives directly from the resolving power of $\mathrm{HU}$, which is the equivalent of (higher-order) decorrelation in SOFI. ${ }^{24}$ SRIR with IPLS allows for a very high-resolution visualization of these results. Whether this visualization is accurate depends to a large degree on the accuracy of the forward operator $\overline{\mathbf{H}}$ in Eqs. (6), (I2), and (I3b).

The largest scale (spatial) effect occurring on sampling that is unaccounted for in the forward model Eq. 12 (including errors in the system response function estimation) limits the accuracy of estimating each of the individual concentration maps shown in Fig. 4. This highlights that features with variations on smaller scales will not be accurately resolved by IPLS on a single concentration map, and likewise on a single channel image. This also impacts on the remixed high-resolution single channel in Fig. 5, despite their appealing appearance.

Resolving two objects from different spectral components, however, is unaffected by this limitation of IPLS, as they are already resolved during the $\mathrm{HU}$ stage. The limited accuracy of IPLS thus only limits the visualization of the $\mathrm{HU}$ resolution gain not the resolving power of $\mathrm{HU}$ per se. We can thus safely claim to have resolved two objects separated $14 \mathrm{~nm}$, or even less (see Fig. 6b), but we cannot claim to know their shapes with the same accuracy. 
Using an AFM image of the same sample, shown in Fig. 4b of previous work, ${ }^{12}$ as a partial truth, suggests there is very reasonable agreement when compared to the images in Fig. 5.

\section{Conclusion}

A new BLHU technique, $\mathrm{BH}$, has been introduced as a variant of rotation of principal components, or an extension of geometric unmixing approaches to data that is not sum-toone constrained, depending on the point of view.

Combining BLHU with $\mathrm{BH}$ and SRIR using IPLS within the HyRes framework has resulted in a minimum resolvable distance of less than $14 \mathrm{~nm}$ on the processed Raman data. This is more than a factor 8 better than the resolution of I I $3 \mathrm{~nm}$ achieved by single channel image-based SRIR with IPLS on the same data, and about a factor 25 better than the intrinsic resolution limit of the Raman microscope. ${ }^{12}$

At the heart of these results lies the resolving power of $\mathrm{HU}$ provided by the validity of Eq. 5. A generalization of HyRes to measurement techniques in which Eq. 5 is violated, such as infrared or fluorescence spectroscopy, will be addressed in a future publication.

\section{Declaration of Conflicting Interests}

The author(s) declared no potential conflicts of interest with respect to the research, authorship, and/or publication of this article.

\section{Funding}

This work has received funding from the European Union's Horizon 2020 research and innovation program under the Marie Skłodowska-Curie grant agreement No. 642742.

\section{ORCID iDs}

Dominik J. Winterauer (D) https://orcid.org/0000-0002-0 I I7-258X Daniel Funes-Hernando (D) https://orcid.org/0000-0002-9177-0160 Bernard Humbert (D) https://orcid.org/0000-000I-6769-2329

\section{Notes}

$\dagger 0.61 \lambda / N A \sim 382 \mathrm{~nm}$ and $0.44 \lambda / N A \sim 275 \mathrm{~nm}$ for $\lambda=532 \mathrm{~nm}$ and $N A=0.85$

$\dagger$ Concentration maps are referred to as abundance maps in remote sensing.

$\S i, j, k$ are matrix/tensor indices.

it Although the quality of the simplex might suffer from dimension reduction if the projection of one or more constituent spectral signatures onto the reduced space is very low.

t. ${ }^{\circ}$ denotes the Hadamard product.

\section{Supplemental Material}

The supplemental material mentioned in the text, consisting of equations and figure, is available in the online version of the journal.

\section{References}

I. D.A. Long. The Raman Effect. Hoboken: John Wiley and Sons, 2002.
2. S. Webster, D.N. Batchelder, D.A. Smith. "Submicron Resolution Measurement of Stress in Silicon by Near-Field Raman Spectroscopy". Appl. Phys. Lett. 1998. 72(12): 1478-1480.

3. J. Grausem, B. Humbert, M. Spajer, et al. "Near-Field Raman Spectroscopy". J Raman Spectrosc. 1999. 30(9): 833-840.

4. R.M. Stöckle, Y.D. Suh, V. Deckert, et al. "Nanoscale Chemical Analysis by Tip-Enhanced Raman Spectroscopy". Chem. Phys. Lett. 2000. 3|8(I-3): $|3|-\mid 36$.

5. E.J. Titus, M.L. Weber, S.M. Stranahan, et al. "Super-Resolution SERS Imaging Beyond the Single-Molecule Limit: An Isotope-Edited Approach". Nano Lett. 2012. I2(10): 5103-5II0.

6. L. Langelddecke, P. Singh, V. Deckert. "Exploring the Nanoscale: Fifteen Years of Tip-Enhanced Raman Spectroscopy". Appl. Spectrosc. 2015. 69(12): |357-137|.

7. A. d'Orlando. Nano-structuration de nanoparticules métalliques pour exaltation de champs électromagnétiques locaux en spectroscopie Raman: Interactions entre champs électromagnétiques localisés sublongueur donde et molécules/particules nano-structurées en champ proche optique. [PhD Thesis]. Nantes, France: University of Nantes, 2015.

8. A. dOrlando, M. Bayle, G. Louarn, et al. "AFM-Nano Manipulation of Plasmonic Molecules Used as Nano-Lens to Enhance Raman of Individual Nano-Objects”. Materials. 2019. 12(9): 1372.

9. L. Duponchel, P. Milanfar, C. Ruckebusch, et al. "Super-Resolution and Raman Chemical Imaging: From Multiple Low Resolution Images to a High Resolution Image”. Anal. Chim. Acta. 2008. 607(2): 168-I75.

10. M. Tomita, H. Hashiguchi, T. Yamaguchi, et al. "Super-Resolution Raman Spectroscopy by Digital Image Processing". J. Spectrosc. 2013. 2013: 459032 .

II. H. Cui, W. Zhao, Y. Wang, et al. "Improving Spatial Resolution of Confocal Raman Microscopy by Super-Resolution Image Restoration”. Opt. Express. 2016. 24(I0): I0767-10776.

12. D.J. Winterauer, D. Funes-Hernando, J.L. Duvail, et al. "Sub-Micron Spatial Resolution in Far-Field Raman Imaging Using PositivityConstrained Super-Resolution". Appl. Spectrosc. 2019. 73(8): 902-909.

13. M. Offroy, M. Moreau, S. Sobanska, et al. "Pushing Back the Limits of Raman Imaging by Coupling Super-Resolution and Chemometrics for Aerosols Characterization". Sci. Rep. 2015. 5: 12303.

14. M.B. Richman. "Rotation of Principal Components". J. Climatol. 1986. 6(3): 293-335.

15. I.T. Jolliffe. Principal Component Analysis and Factor Analysis. New York, NY: Springer New York, 2002. Chap. I I, Pp. 269-298.

16. M.D. Craig. "Minimum-Volume Transforms for Remotely Sensed Data". IEEE Trans. Geosci. Remote Sens. 1994. 32(3): 542-552.

17. W.K. Ma, J.M. Bioucas-Dias, T.H. Chan, et al. "A Signal Processing Perspective on Hyperspectral Unmixing: Insights from Remote Sensing". IEEE Signal Process. Mag. 20I4. 3I(I): 67-8I.

I8. E. Chouzenoux, M. Legendre, S. Moussaoui, et al. "Fast Constrained Least Squares Spectral Unmixing Using Primal-Dual Interior-Point Optimization". IEEE J Sel. Top. Appl. Earth Obs. Remote Sens. 2014. $7(1): 59-69$.

19. T. Akgun, Y. Altunbasak, R.M. Mersereau. "Super-Resolution Reconstruction of Hyperspectral Images". IEEE Trans. Image Process. 2005. I4(II): 1860-1875.

20. M.J. Ehrhardt, K. Thielemans, L. Pizarro, et al. "Joint Reconstruction of PET-MRI by Exploiting Structural Similarity". Inverse Probl. 2014. 3I(I): $01500 \mathrm{I}$.

2I. L. Loncan, L.B. de Almeida, J.M. Bioucas-Dias, et al. "Hyperspectral Pansharpening: A Review". IEEE Geosci. Remote Sens. Magazine. 2015. 3(3): 27-46.

22. C. Lanaras, E. Baltsavias, K. Schindler. "Hyperspectral SuperResolution by Coupled Spectral Unmixing". In: Proceedings of the 2015 IEEE International Conference on Computer Vision (ICCV). December 2015. Santiago, Chile: 7-13 2015. Pp. 3586-3594. 
23. L. Bungert, D.A. Coomes, M.J. Ehrhardt, et al. "Blind Image Fusion for Hyperspectral Imaging with the Directional Total Variation". Inverse Probl. 2018. 34(4): 044003.

24. T. Dertinger, R. Colyer, G. lyer, et al. "Fast, Background-Free, 3d Super-Resolution Optical Fluctuation Imaging (SOFI)". Proc. Natl. Acad. Sci. U.S.A. 2009. 106(52): 22287-22292.

25. D. Funes-Hernando, M. Pelaez-Fernandez, D.J. Winterauer, et al. "Coaxial Nanowires as Plasmon-Mediated Remote Nanosensors". Nanoscale. 2018. 10(14): 6437-6444.

26. A. de Juan, R. Tauler. "Chemometrics Applied to Unravel Multicomponent Processes and Mixtures: Revisiting Latest Trends in Multivariate Resolution”. Anal. Chim. Acta. 2003. 500(I): 195-210.

27. S.C. Park, M.K. Park, M.G. Kang. "Super-Resolution Image Reconstruction: A Technical Overview". Signal Process. Mag. IEEE. 2003. 20(3): $2 I-36$.

28. A. de Juan, J. Jaumot, R. Tauler. "Multivariate Curve Resolution (MCR). Solving the Mixture Analysis Problem". Anal. Methods. 2014. 6 : 4964-4976.

29. N. Keshava. "A Survey of Spectral Unmixing Algorithms". Lincoln Lab. J. 2003. I4(I): 55-78.

30. J.M. Bioucas-Dias, A. Plaza, N. Dobigeon, et al. "Hyperspectral Unmixing Overview: Geometrical, Statistical, and Sparse RegressionBased Approaches". IEEE J Sel Top. Appl. Earth Obs Remote Sens. 2012. 5(2): 354-379.

3I. K. Pearson. "LIII. On Lines and Planes of Closest Fit to Systems of Points in Space". London Edinburgh Dublin Philos. Mag. J. Sci. 1901. 2(II): 559-572.

32. C. Eckart, G. Young. "The Approximation of One Matrix by Another of Lower Rank". Psychometrika. 1936. I(3): 2II-2I8.

33. I.T. Jolliffe, J. Cadima. "Principal Component Analysis: A Review and Recent Developments". Philos. Trans. R. Soc., A. 2016. 374(2065): 20150202.

34. R. Bro, A.K. Smilde. "Principal Component Analysis". Anal. Methods. 20|4. 6(9): 28I2-283।.

35. J.M. Craddock, C.R. Flood. "Eigenvectors for Representing the $500 \mathrm{mb}$ Geopotential Surface Over the Northern Hemisphere". Q.J.R. Meteorol. Soc. 1969. 95(405): 576-593.

36. S.A. Farmer. "An Investigation into the Results of Principal Component Analysis of Data Derived from Random Numbers". J. R. Stat. Soc. Ser. D (The Statistician). 197I. 20(4): 63-72.

37. M.E. Winter. "N-FINDR: An Algorithm for Fast Autonomous Spectral End-Member Determination in Hyperspectral Data". In: M.R. Descour and S.S. Shen (Eds.) Imaging Spectrometry V. USA: International Society for Optics and Photonics, 1999, Vol. 3753. Pp. 266-276.

38. J.M. Nascimento, J.M. Dias. "Vertex Component Analysis: A Fast Algorithm To Unmix Hyperspectral Data". IEEE Trans. Geosci. Remote Sens. 2005. 43(4): 898-910.
39. J.M. Bioucas-Dias. "A Variable Splitting Augmented Lagrangian Approach to Linear Spectral Unmixing". In: First Workshop on Hyperspectral Image and Signal Processing: Evolution in Remote Sensing, 2009. WHISPERS'09. IEEE, USA. Grenoble, France: 26-28 August 2009. Pp. I-4.

40. C.H. Lin, C.Y. Chi, Y.H. Wang, et al. "A Fast Hyperplane-Based Minimum-Volume Enclosing Simplex Algorithm for Blind Hyperspectral Unmixing.". IEEE Trans. Signal Process. 2016. 64(8): 1946-1961.

4I. P. Yin, Y. Sun, J. Xin. "A Geometric Blind Source Separation Method Based on Facet Component Analysis". Signal Image Video Process. 2016. 10(I): 19-28.

42. J. Zhao, H. Lui, D.I. McLean, et al. "Automated Autofluorescence Background Subtraction Algorithm for Biomedical Raman Spectroscopy”. Appl. Spectrosc. 2007. 6I(II): I225-1232.

43. J. Duvail, P. Retho, S. Garreau, et al. "Transport and Vibrational Properties of Poly (3,4-ethylenedioxythiophene) Nanofibers". Synth. Met. 2002. I3I(I-3): I23-I28.

44. L.L. Thurstone. "A New Rotational Method in Factor Analysis". Psychometrika. 1938. 3(4): 199-218.

45. A.A. Green, M. Berman, P. Switzer, et al. "A Transformation for Ordering Multispectral Data in Terms of Image Quality with Implications for Noise Removal". IEEE Trans. Geosci. Remote Sens. 1988. 26(I): 65-74.

46. J.M. Bioucas-Dias, J.M.P. Nascimento. "Hyperspectral Subspace Identification". IEEE Trans Geosci. Remote Sens. 2008. 46(8): 2435-2445.

47. R. Tauler, A. Izquierdo-Ridorsa, E. Casassas. "Simultaneous Analysis of Several Spectroscopic Titrations with Self-Modelling Curve Resolution”. Chemom. Intell. Lab. Syst. 1993. 18(3): 293-300.

48. P. Paatero, U. Tapper. "Positive Matrix Factorization: A Non-Negative Factor Model with Optimal Utilization of Error Estimates of Data Values". Environmetrics. 1994. 5(2): III-126.

49. R. Bro, S. De Jong. "A Fast Non-Negativity-Constrained Least Squares Algorithm". J. Chemom. 1997. II(5): 393-40I.

50. D.D. Lee, H.S. Seung. "Learning the Parts of Objects by Non-Negative Matrix Factorization”. Nature. 1999. 40I(6755): 788.

5I. P.O. Hoyer. "Non-Negative Matrix Factorization with Sparseness Constraints". J. Mach. Learn. Res. 2004. 5(Nov): |457-| 469.

52. S. Moussaoui, D. Brie, A. Mohammad-Djafari, et al. "Separation of Non-Negative Mixture of Non-Negative Sources Using a Bayesian Approach and MCMC Sampling”. IEEE Trans Signal Process. 2006. 54(II): 4|33-4I45.

53. J. Li, J.M. Bioucas-Dias. "Minimum Volume Simplex Analysis: A Fast Algorithm to Unmix Hyperspectral Data". In: IEEE International Geoscience and Remote Sensing Symposium, 2008. IGARSS 2008. Vol. 3. IEEE, USA. Boston, MA, USA: 7-II July 2008. Pp. III-250-III253. 